\title{
Distorting Social Feedback in Visualizations of Conversation
}

\author{
Tony Bergstrom and Karrie Karahalios \\ University of Illinois \\ \{abergst2,kkarahal\}@cs.uiuc.edu
}

\begin{abstract}
Real-time collaborative visualization of conversation affects small group dynamics and encourages balanced participation. Visual feedback plays the role of a gentle moderator encouraging a speaker to yield the floor. To better evaluate the effect of visual feedback on collaboration, we purposefully distorted the apparent balance in the Conversation Clock, a shared visualization of conversation. We present a pilot study examining various distortion strategies followed by a more in depth study applying distortion to discussion. Our results indicate that participants will accept some significantly distorted visualizations as an accurate representation of conversation; however, distorted feedback provides only minimal impact on conversational balance. These findings suggest the mechanism in visualization that drives individuals towards balanced conversation is not solely influenced by the visual feedback and remains an open question for future work.
\end{abstract}

\section{Introduction}

Shared real-time visualizations of group conversation influence the behavior of individuals in small groups. Numerous researchers have designed visualizations depicting a speaker's contribution in face-to-face co-located interaction $[1,2,9,24,25,33]$. While the purposes of these projects vary from mediating a meeting, to providing anonymous feedback, to generating more ideas; studies have repeatedly shown that visualizations of face-to-face participation encourage a balanced conversation. This change in dynamics is not entirely unexpected as people purposefully control their appearance and mannerisms to project a certain persona to others [17]. Visualizations of conversation publicly display characteristics that one may desire to control and change in the context of the current group, such as pulling back from dominating conversation when with a group of friends. Anecdotally, people want to appear engaged but do not want to appear to talk the entire time, and they use the visualization to evaluate themselves. These visualizations can be collectively called Social Mirrors as they provide an unbiased, third-person, real-time perspective on social information in much the same way that a traditional mirror reflects a third person real-time perspective of visual information. Capturing ephemeral social interaction, these Social Mirrors reveal perspectives that one might otherwise miss [2].

This work investigates a more nuanced view of the social mirror's influence on group dynamics. Specifically, we contrast the use of accurate feedback versus distorted feedback by using the Conversation Clock to distort the feedback by indicating a participant spoke much more than in reality [2]. In this paper, we first demonstrate four distortion strategies and their effectiveness at misleading the viewer before applying them to group conversations. In a further study, we show that people trust and accept a significantly distorted social mirror as a representation of conversation. However, only a small change in participation can be attributed to this distortion. In dyadic conversation, a 2-second per minute change in participation was measured from a $160 \%$ distortion of contribution. We conclude that the pressure a social mirror applies is not directly tied to the accuracy of the visualization it displays.

In the following sections, we describe the theory and two studies using a social mirror. We conclude by discussing the implications for social visualization and regulation of group dynamics.

\section{Visual Signals}

Computer-mediated communication research strives to improve and explore the cues we send and receive in new environments and interfaces. Particularly in video-based chat, visual signals often replicate face-to-face interaction. These signals include body language, facial expressions, eye contact, and gestures - feedback that is desirable but often lacking in traditional remote conversation $[4,21,29]$. In a collocated environment, producing the same feedback is less desirable as it duplicates the existing feedback.

Other literature features abstracted visual imagery to improve remote interaction. This imagery can range from all-encompassing virtual environments, to social visualizations, to activity indicators $[10,11,13,23$, 30]. As in avatar enabled virtual worlds, this imagery provides a grounded environment that sets the rules on how to interact and what is allowed. In many cases, 
information is presented in a novel visualization that reveals interaction patterns that are otherwise not available. These types of signals are useful in remote environments, but they can also add information to the face-to-face environment by signaling activity, dominance, and history that is otherwise nonexistent in faceto-face conversation.

Applying similar abstracted feedback in face-toface conversation influences group dynamics by encouraging and discouraging participation [1, 2, 9, 24]. DiMicco et al. examined visualization as a way to encourage idea generation through discussion. The shift toward a balanced conversation was seen as as result of the visualization [9]. The group continued studying the effects of real-time and post-meeting visualizations and found these visualizations encourage informationsharing behavior [7].

Similar to DiMicco, work by Bachour et al. and Bergstrom et al. further examined balance in settings with integrated displays $[1,2]$. Both groups used the table as the display either by embedded lights or overhead projection. Both visualizations balanced conversation. The Conversation Clock visualization differed from previous work in that it depicted subtle changes in turn-taking behavior. Conversation dynamics are apparent as the visualization showed overlapping speech and three visual samples per second. Each individual speaker is represented with a different color. As a result, it showed how behavior differed in people who were talkative from those were generally quiet. Work by Kim et al. tested a handheld feedback device that provided similar conversation feedback in addition to sensing features of speech, body movement, proximity, etc [24]. Their work further demonstrated the use of group dynamic feedback in remote scenarios has a similar effect. Anecdotal evidence from these groups suggests the details of the visualization had an effect; however, the changes can be attributed to the presence of the visualization as easily as to the feedback from it.

Others have used visualization to support or discourage specific interactions outside of contribution. Leshed et al. demonstrated that visual feedback based on language can encourage more positive language in text-based chat environments [27]. The public awareness and resulting accountability for negative comments discouraged overly negative criticism. Others have worked to redirect both positive and negative feedback into anonymous backchannels by allowing participants to voice their opinion via real-time voting $[3,20]$. The explicit positive and negative feedback uses the visualization to show the group's aggregate approval. Participants using the visualizations reported increased satisfaction with the interaction when their feedback was publicized.

\section{Social Self}

Our work centers around Goffman's theory of "face" [17] as a means to provide social influence and change interaction. Establishing a good face is a combination of revealing and hiding information to produce a positive persona in the minds of others. Social signals such as actions, words, and gestures all influence one's face. A person chooses appropriate responses to fit the context and social protocol. For example, a sales clerk might always choose to convey a polite and helpful demeanor by offering help, smiling, and showing respect to the customer. These signals are not always trustworthy. The same clerk might be frustrated but hide that frustration behind a smile and polite words to fulfill his role. The proper face keeps the clerk employed and the customer satisfied. However, many unconscious cues can reveal emotions of which we are unaware $[12,28]$. An astute customer might notice and incorporate this knowledge into their further interactions.

Goffman's work on maintaining face also applies in the digital domain. Research on social network sites demonstrates the active control of digital cues to present a desired image. In the dating world, these cues might be purposefully misleading in representing height, weight, age, and interests to attract a certain type of person [18]. More general audiences in social networks provide cues through posted pictures, favorite music, movies, books, and recent activities to friends and acquaintances $[8,26]$. The degree to which a person manages their identity varies by personality and goal. For example, whereas the average college student might be more open to sharing pictures and personal comments, a person seeking a job might choose to remain more professional in public dialog.

These subtle aspects of presentation unconsciously influence how other people choose to interact in response and thus how they appear to others. Accommodation theory suggests individuals converge on a predictable interaction style during an exchange. In friendly circumstances, people will adopt similar sentence grammar, accent, tone, word choice, etc to establish a cohesive group [16, 34]. Conversely, differences in the same cues, such as emphasizing accent or word choice, show social distance by indicating the other is not a part of the in-group.

Increasingly, empirical work has applied technology to automatically model and detect the nuances of conversation activity [14]. This work can be categorized area into topics of capturing, understanding, and predicting subtle patterns in interaction. These works have focused on broad categories such as modeling interaction management through turn taking and addressing; inferring internal states showing interest, anxiety, embarrassment, boredom, etc.; detecting per- 
sonality traits of dominance and extroversion; and understanding roles in conversation.

To present the best face, a person must be aware of their own signals. Goffman commonly used the metaphor of a theater wherein individuals must work to convey the appropriate characters to the audience, no matter what occurs on stage. Technology is serving to reveal a peek of the backstage area.

\section{Conversation Clock}

This work builds upon the Conversation Clock tabletop visualization by contrasting the effect of accurate and distorted visualizations of vocal contribution. In this section we briefly overview the interface and discuss the distortion strategies we explored.

The Conversation Clock visualizes conversational speech by monitoring people around a table. Dedicated lapel microphones identify each participant's contribution which is then rendered onto the tabletop as a series of rectangles. The rectangle's color indicates speaker while the length indicates volume. The rectangles appear along a concentric circular timeline, each ring capturing one minute of time (Figures 1 and 2). The outermost ring shows the most recent conversation while completed rings are compressed, in a brief animation, toward the center of the table. Throughout the conversation, simultaneous speech appears as overlapping rectangles with the quieter participant represented by a smaller rectangle. For the purpose of later analysis, we define a lead as a moment when a person is the loudest speaker, whether simultaneous or solo.

Qualitative feedback from prior studies indicates that the Conversation Clock affects the talkative individuals more than the quiet ones [2, 22]. Participants cite salient patterns in turn-taking and conversation dominance as the motivations to change. Our distortion strategies seek to emphasize these patterns to have a greater effect.

\subsection{Distortion Strategies}

We distorted the Conversation Clock to make people appear more talkative and dominating. We chose to increase the number of salient moments in which participants notice themselves dominating conversation. The Conversation Clock will actively produce more of their representative color or make it more noticeable.

We began with a list of four potential targets for distortion strategies to test in the pilot study: amplitude (volume), speed (sample rate), color brightness of rectangles, and color replacement in past samples (i.e. swapping the color of rectangles from other participants' assigned colors to the emphasized person's color). Low, Medium, and High conditions of each strategy were tested. Descriptions of each strategy ap-

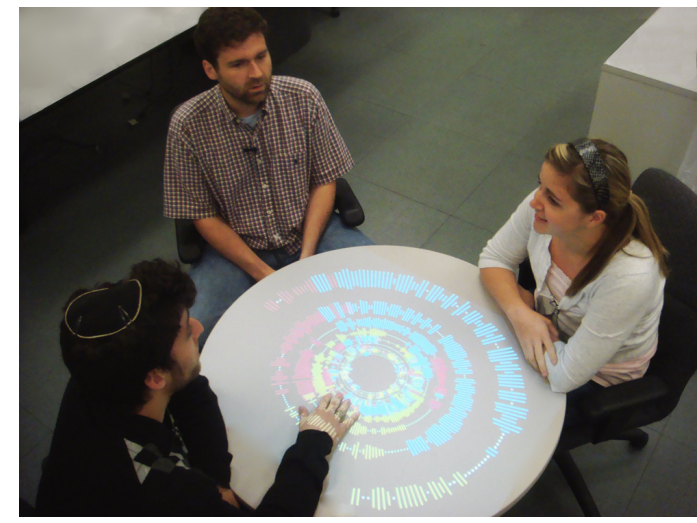

Figure 1. The Conversation Clock captures conversation via microphones to demonstrate patterns of interaction. Past studies have shown that collocated conversation tends to balance in response to the visualization's presence. In this work, we appropriate the visualization for remote use to examine the effects of a distorted visualization on conversation.
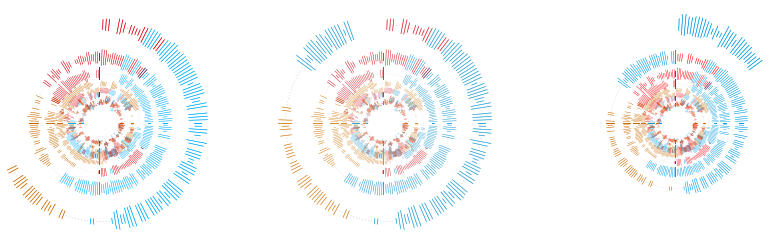

Figure 2. The Conversation Clock depicts contributions to conversation as bars progressing around a circle. Color indicates speaker, bar amplitude indicates volume, overlapping colors show overlapping speech. Each minute of conversation is captured as a ring with the outermost ring indicating the most recent activity.

pear in the following paragraphs; simplified renderings are shown in Figure 3.

Speed changes the sampling rate of the Conversation Clock. When the emphasized person leads the conversation, the sample rate increases and more bars are drawn for this person. Low through High conditions vary the sample rate to a lower and higher frequency, respectively.

Amplitude increases the length of the speaker's rectangular bars. This implies the speaker was louder throughout the conversation. Low through High Conditions vary the amplitude multiplier with low being a slight increase to high being the largest increase in size. 


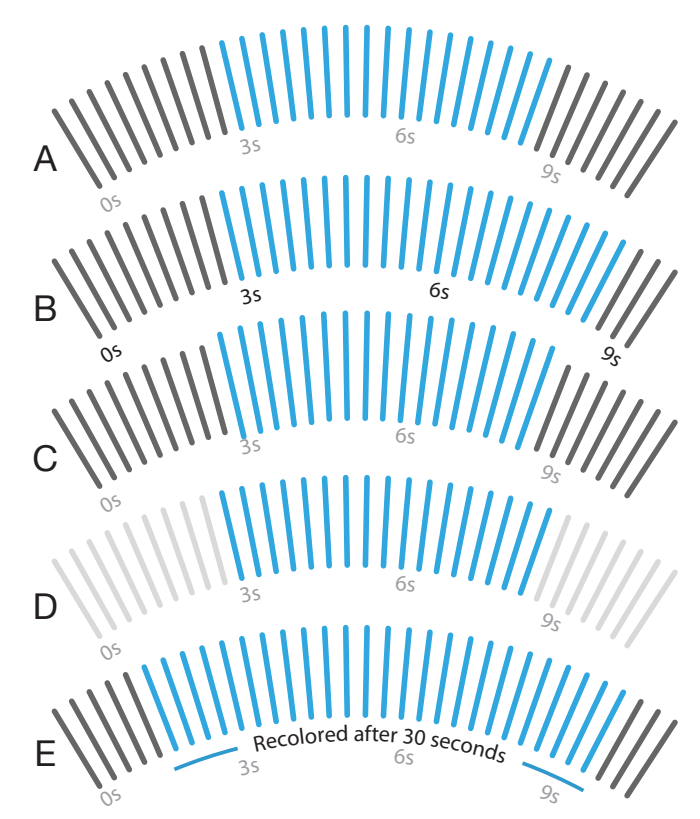

Figure 3. For the same conversation sample we show equivalent example renderings for the blue speaker under (A) Normal, (B) Speed Emphasis, (C) Amplitude Emphasis, (D) Brightness Emphasis, (E) Color Replacement Emphasis.

Brightness emphasizes one participant by decreasing the color brightness of all other participants. Low through High Conditions vary the percent of reduction in brightness with low being a subtle difference to high being the largest difference in brightness.

Color Replacement changes the rendering of the person speaking in previously drawn history. The emphasized person's color is used to recolor its neighbors 30 seconds after they are drawn. Low through High conditions vary the number of neighboring bars affected: a low condition will affect the two nearest bars on both sides whereas the high condition changes four bars on both sides.

The distortion techniques leverage natural deficiencies in visual cognition such as change blindness and awareness blindness [31, 32]. People cannot attend to all of their surroundings concurrently. Therefore, many of the changes remain unnoticed. In this setting, participants attend primarily to the conversation, their own speech, and the table visualization. They are less likely to notice the distortion as it happens. In the case of color replacement, they do not notice the distortion as in changes right in front of them. An untrusted visualization is likely to be disregarded as wrong, we designed the graphical distortions to be subtle and trusted in the short term, yet largely suggestive of dominant participation in the long term.
For our later analysis, we label participants "overemphasized" when they are targets of a distortion strategy. Their contribution is over-represented in the visualization. In the same condition, their discussion partner is labeled "underemphasized" as their contribution is visually lessened as a portion of conversation.

\section{Experimental Studies}

To investigate the effect of distortion on face-toface conversation participation, we designed two studies. The first is a pilot study to evaluate the distortion strategies. Participants viewed a pre-recorded conversation alongside corresponding Conversation Clock visualization distortion. From this study we determined which conditions were effective at misrepresenting balance while still engendering trust that the visualization was an accurate depiction of the spoken conversation. The second study was a remote conversation to remove all other visual cues from conversation and focus attention primarily on the visualization. In this study, we show that social mirrors can be inaccurate while influencing a shift towards participation balance.

\subsection{Hypotheses}

We began with the following hypotheses:

H1 A speaker will perceive they are contributing more when their contribution is emphasized via distortion. People will defer to the visualization to gauge their participation in conversation rather than trusting their instinct or memory.

H2 Overemphasizing a speaker's contribution decreases participation from that speaker. Prior work indicates talkative people are conscious of appearing to dominate the conversation and specifically cited the visualization's role in their behavioral change.

H3 When emphasized, people will report feeling pressure to speak less due to the public feedback. In prior work, awareness of the visualization affected conversation. With distortion applied to an individual, the same awareness of dominating conversation should happen sooner and more often. In addition to changing behavior in $\mathrm{H} 2$, we expect that that the increased dominance will manifest as a social pressure attributed to the visualization.

\section{Pilot Study}

We began by testing the four distortion strategies to narrow the experimental conditions for the larger study. We predicted our intended strategy would lead a viewer to overestimate the amount of time the distorted individual spoke without the viewer being aware of 
that distortion. For this pilot study, our selection of distortion strategy was chosen based upon participants' written estimations of participation and Likert scale feedback.

Participants were presented with 4.5-5 minute videos of recorded conversations selected from interview segments of "The Daily Show" (a popular news entertainment program) while the Conversation Clock visualization was projected synchronously on the table. The video was displayed on a screen opposite the participant. Prior to the study, each video was hand-coded for speaker participation to provide the volume and turntaking parameters for the Conversation Clock. The interviewer or the interviewee from the video clips were randomly selected to be overemphasized by distortion.

Participants observed a total of 13 total conditions: a Low, Medium, and High condition for each of the four distortion strategies and one control condition. The Low condition was a subtle distortion and would only be noticed if one paid close attention. The High condition was extremely distorted and it was easy to notice they were distorted if the observer knew they were being distorted. The Medium condition fell in the middle. The conditions were randomly shuffled, however the High conditions were never adjacent.

At the end of each condition, the study participants estimated the proportion of time each person spoke during the conversation and indicated the accuracy of the Conversation Clock's depiction of conversation. The variable, Overestimation, was measured as the percent error relative to the distorted person's speaking time. Eight people participated in the pilot study (5 males, 3 females). They were told all visualizations would differ, though the distortion strategies were not revealed until after the study. The session lasted approximately 1.5 hours, participants were remunerated with gift certificates to Amazon.com.

\subsection{Pilot Study Results}

Figure 4 shows the results of the participant estimations as reported in the surveys. All four strategies showed a tendency to deviate from the control condition estimates. We examined the conditions that maximized overestimation for each strategy and found that they differ from the control condition via pairwise ttests: (High Amplitude, High Speed, Medium Brightness, and Medium Color Replacement). All paired ttests showed significance $(\mathrm{p}<0.04)$ : with our small sample of 8 individuals the effect size is large enough to indicate the conditions were effective.

Aggregate Likert scale data indicated that no distortion strategies were misleading. Individuals voiced a few concerns that varied over the conditions. With the amplitude strategy, some participants remarked "the bars seem bigger" while in the brightness condition

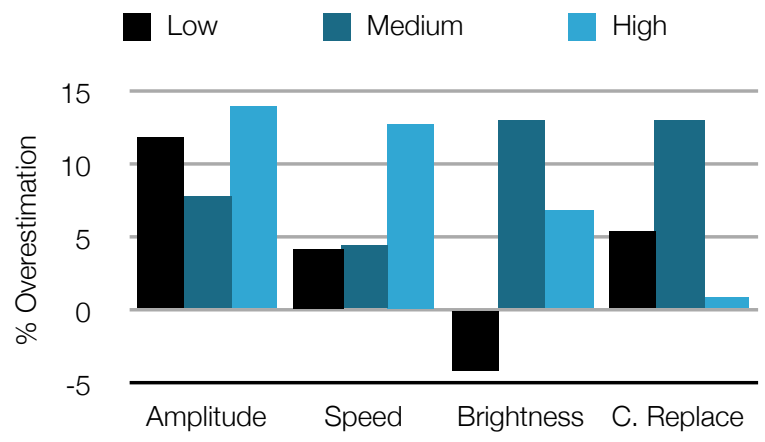

Figure 4. The results of the pilot study indicate all strategies encouraged overestimation with at least one setting (Low, Medium, High). The highest bar in each represents a significant deviation from the control.

some participants noted, "It's harder to see [the underemphasized speaker's] color." The High Color Replacement condition was the only condition to illicit any extreme distrust vocally; a single individual commented "I don't know how, but it's wrong. It's just wrong." He further explained that he had explicitly watched the bars as they appeared to determine what was going on, and he found no error, but refused to trust it.

Aside from individual exceptions, the pilot study demonstrated these distorted social mirrors were trusted and accepted as accurate in a real time situation. In choosing the strategy to pursue for the remote conversation study, we chose to initially test the strategies in some group conversations. These test groups highlighted aspects we hadn't considered initially. For example, Amplitude, one of the best performing distortion strategies, had to be eliminated. People were consistently aware of the amplitude distortion when it was their own speech that was being distorted. They attributed the distortion to a miscalibrated microphone. They stopped the conversation so they could adjust the microphone or ask the experimenter to check the audio controls. Brightness was eliminated for similar reasons; people thought the projector was not displaying color properly or that the researchers made a bad choice in colors. We chose to pursue Speed as a distortion because it provided immediate visual feedback and never received any negative criticism.

\section{Conversation Study}

The conversation study examined how distorted feedback could affect balance in actual conversation. For the conversations, we choose to focus attention on the visualization by utilizing a remote conversation setting. We did this to encourage participants to view the visual distortion in the Conversation Clock without 
external distraction. By placing individuals in separate rooms, traditional visual cues were eliminated. Each participant viewed the Conversation Clock on a monitor in front of them; each participant saw identical visualizations.

We began each session by explaining the Conversation Clock visualization. Then the participants were given time to familiarize themselves with the colors, time structure, and animation by introducing themselves to each other. Following the introduction, a full session consisted of four 15-minute conditions between two individuals. Conditions included a no visualization condition (No Vis), a traditional Conversation Clock visualization, a condition in which one person was distorted, and a condition in which the other person was distorted. Following each conversation, participants answered a set of Likert scale questions. We randomly ordered the visual conditions for each pair. They were not told about the distortion until after the study concluded.

We encouraged conversation using hypothetical questions selected from Gregory Stock's "The Book of Questions." Four sets of three questions were constructed and randomized for use in the study. Participants were free to answer the questions in any order or deviate from questions as a topic progressed. However, we asked that participants start their conversations from these questions to prevent conversations from carrying over between conditions and affecting the results. Two example questions follow:

If you were able to live to the age of ninety and retain either the body or the mind of a thirty year old for the last sixty years of your life, which would you choose?

If you could spend one year in prefect happiness but afterward would remember nothing of the experience would you do so? If not, Why not?

Thirteen groups of two participated in this study (10 male / 16 female). Participants were recruited using flyers posted on campus and via common mailing lists. The final population included both students and staff. Full sessions generally lasted about 1.5 hours. Participants received gift certificates from Amazon.com for their participation.

\subsection{Interaction Measures}

For each person, we recorded the duration of speech, the number of turns taken, the length of those turns, and the degree to which an individual was overemphasized by the distortion strategy. This data was automatically captured and normalized by the Conversation Clock. We also collected the following qualitative data with a Likert scale survey:
Q1 I spoke than others. (Less / More)

Q2 I spoke than usual. (Less / More)

Q3 My partner pressured me to speak more or less. (Agree / Disagree)

Q4 The clock pressured me to speak more or less. (Agree / Disagree)

Q5 My partner was affected by the clock. (Agree / Disagree)

Q6 My partner understood my viewpoint. (Agree / Disagree)

Q7 I understood my partner's viewpoint. (Agree / Disagree)

Q8 The conversation was natural. (Agree / Disagree)

Q9 I was aware of my depiction in the clock. (Agree / Disagree)

\subsection{Conversation Study Results}

This study demonstrates a change in participation as a function of the distortion condition. People adjusted the total amount of their speech when distorted with speed; however, the change was small (Figure 5) compared to the degree of distortion in the conditions. Analysis indicates most of this change occurs early and stabilizes afterwards.

The second column of Table 1 indicates the emphasis condition significantly affected overall contribution to conversation $(\mathrm{F}(2,48)=4.2, \mathrm{p}<0.02)$. Figure 5 makes this effect apparent: emphasized people speak less and underemphasized people speak more. However, the difference is minimal. The aggregate results

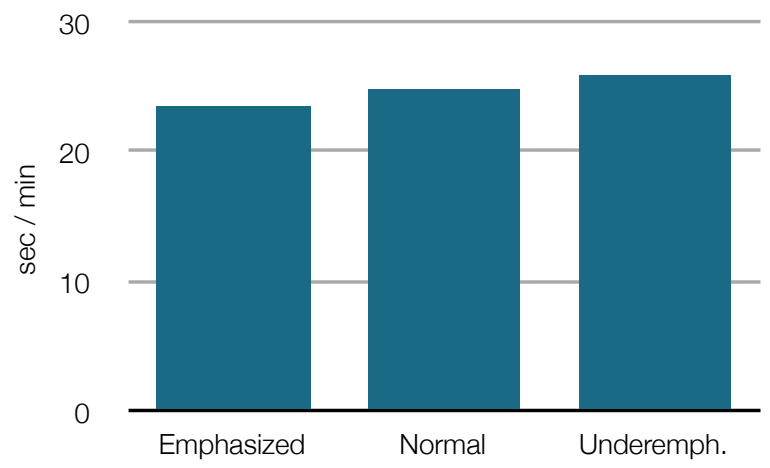

Figure 5. The conversation study demonstrated that emphasis condition played a significant role in contribution to conversation $(F(2,48)=4.2, p<0.02)$. However, the observed change between Emphasized and Unemphasized amounts to only 1.9 seconds per minute. 


\begin{tabular}{rccc} 
Teasure & $F(1,24) p$ val & $\begin{array}{c}\text { Condition } \\
F(2,48)\end{array}$ val & $\begin{array}{c}\text { Cond:Talk/Quiet } \\
F(2,48)\end{array}$ val \\
\hline Leads & $\mathbf{9 . 8} \mathbf{p}<\mathbf{0 . 0 0 5}$ & $\mathbf{4 . 2} \mathbf{p}<\mathbf{0 . 0 2}$ & $0.5 \mathrm{p}<0.6$ \\
Turns & $0.6 \mathrm{p}<0.5$ & $\mathbf{1 6 . 2} \mathbf{p}<\mathbf{0 . 0 0 0 1}$ & $0.7 \mathrm{p}<0.6$ \\
Length & $\mathbf{6 . 4} \mathbf{p}<\mathbf{0 . 0 2}$ & $1.3 \mathrm{p}<0.3$ & $0.5 \mathrm{p}<0.6$ \\
$Q 1$ & $\mathbf{6 . 2} \mathbf{p}<\mathbf{0 . 0 2}$ & $0.8 \mathrm{p}<0.5$ & $0.5 \mathrm{p}<0.6$ \\
$Q 2$ & $1.2 \mathrm{p}<0.3$ & $1.6 \mathrm{p}<0.3$ & $0.2 \mathrm{p}<0.8$ \\
$Q 3$ & $0.2 \mathrm{p}<0.7$ & $0.5 \mathrm{p}<0.7$ & $0.2 \mathrm{p}<0.9$ \\
$Q 4$ & $1.0 \mathrm{p}<0.4$ & $0.4 \mathrm{p}<0.7$ & $0.4 \mathrm{p}<0.7$ \\
$Q 5$ & $2.0 \mathrm{p}<0.2$ & $0.6 \mathrm{p}<0.6$ & $0.8 \mathrm{p}<0.5$ \\
$Q 6$ & $0.5 \mathrm{p}<0.5$ & $0.4 \mathrm{p}<0.7$ & $0.4 \mathrm{p}<0.7$ \\
$Q 7$ & $0.1 \mathrm{p}<0.7$ & $0.0 \mathrm{p}<1$ & $0.3 \mathrm{p}<0.8$ \\
$Q 8$ & $0.0 \mathrm{p}<0.9$ & $0.6 \mathrm{p}<0.6$ & $0.5 \mathrm{p}<0.7$ \\
$Q 9$ & $0.5 \mathrm{p}<0.5$ & $0.1 \mathrm{p}<0.9$ & $1.6 \mathrm{p}<0.3$
\end{tabular}

Table 1. This table shows the results of a repeated measures analysis comparing between conditions, the Talkative/Quiet split, and the two combined.

across all participants show an emphasized individual will speak 2 seconds less in a minute than when in the corresponding underemphasized condition $(\mathrm{t}(25)=2.8$, $\mathrm{p}<0.01)$. This small difference was incurred with the Conversation Clock presenting a contribution of $160 \%$. For every 10 seconds of time an emphasized individual spoke, the speed distortion would render their appearance as 16 seconds of speaking.

Exploring the conversational balance on a minute by minute basis (Figures 6-7), the conditions differ over time $(F(2,1133)=8.166, p<0.003)$. Specifically, the relative balance between emphasized and underemphasized shifts and becomes stable at approximately the sixth minute of conversation. Beyond that, the the two progress in an essentially parallel manner. Figure 6

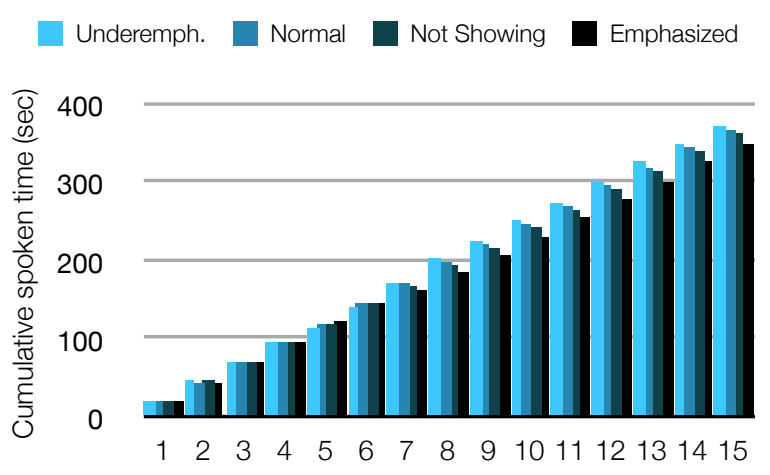

Figure 6. The average contribution under each condition produce generally similar lines. The exception is at minutes 6-8. See Figure 7 for more details. demonstrates deviations in minutes 6-8 relative to all other minutes. For the emphasized condition, these minutes are consistently lower than the rest of the minutes $(t(130)=3.08, p<0.002)$. As groups were given three questions to discuss, minutes 6-8 were a common time to end the first question and move to the second. One hypothesis suggests that at this point, the emphasized speaker has time to observe their depiction in the Conversation Clock during the lull in conversation as they reread the questions to choose the next one.

Though we continued to categorize participants as talkative or quiet as in past work, Table 1 indicates that the Talkative and Quiet participants reacted relatively similarly to the visualization in this setting. Changes in participation were limited to the conditions and not the individuals. For example, the number of turns consistently increased when a person was emphasized $(\mathrm{F}(2,48)=16.2, \mathrm{p}<0.0001)$ with each person taking an additional 6.2 turns per minute, up from 5.8 turns in a normal condition.

In spite of the focus on the visualization and removal of other conversational cues, our participants did not report notable pressure from the visualization or from their partner. There were changes in interaction, specifically in the length of turns. Though they provide a slight change in interaction, the distortions in the social mirror reman relatively unnoticeable.

\subsection{Discussion}

Distorted visualizations impacted the perception of behavior. In both the pilot study and this study participants accepted the visualization feedback as a faithful depiction of conversation. Combining results from this study and the pilot, we were able to validate $\mathrm{H} 1$ : a speaker will perceive they are contributing more when their contribution is emphasized via distortion. In the pilot study, each of the distortion strategies skewed the viewer's estimation of speech contribution.

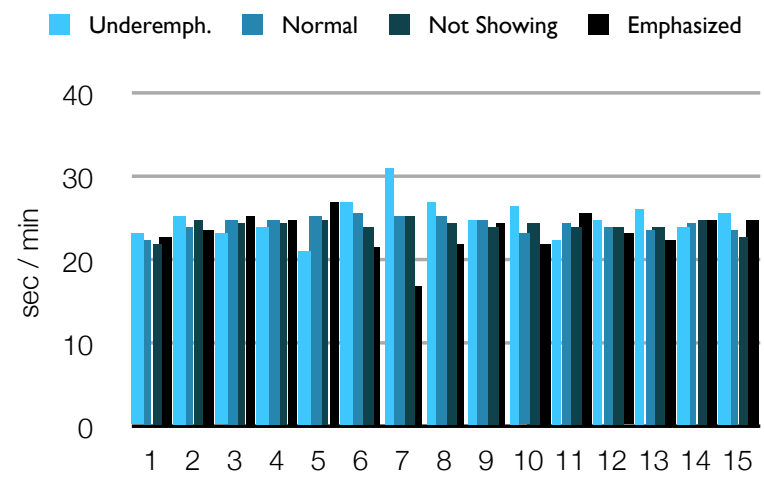

Figure 7. A minute by minute look at the contribution of participants shows that most of the difference in conditions occurs at minutes 6-8. 
With H2 we had hypothesized that overemphasizing a person would decrease that speaker's participation. The second study validates our hypothesis, though with a small aggregate difference of a few seconds per minute.

Interestingly, as seen in Figure 7, the most salient moment of change consistently occurs roughly 6 minutes into conversation. This uniformly corresponds with the first major question transition. Most pairs chose to answer one question at a time and discuss it in depth before moving on. That transition between questions seems to be when most attention was paid to the visualization and where the balance converged for the remainder of the conversation. After that point in conversation, we saw very little in terms of changes in balance (Figure 6). Similar to what Gersick termed a midpoint transition, groups used a landmark in conversation to set the pacing for further discussion [15]. However, once this first insight was made, there is very little aggregated change.

As in prior work, we did not detect any differences between the Talkative and Quiet participants. We expect that dyadic conversations impacted the types of results we could detect due to the dynamics of two person conversation $[5,19]$. A two person conversation has only a speaker and a listener, there is no third or fourth person to share the speaking load in conversation. The lack of contrasts between talkative and quiet participants can be attributed to this change. In a dyad, one cannot remain quiet and still be a part of conversation. The only distinctions made between the talkative and quiet in this study is that the two groups were appropriately labeled: the talkative consistently dominated measures of Leads, Turns, and Turn Length throughout.

H3 stated that emphasized people will report feeling pressure to speak less due to the public feedback. Measured primarily through our Likert scale questions, participants made no indications of additional pressure. We could not prove or disprove this hypothesis. It is not clear whether the emphasis distortions as rendered in the visualization provided any additional pressure to change one's behavior since the resulting change in participation was itself so small.

\section{Implications and Conclusion}

People will trust and accept a distorted image of their interaction. They will react to their distorted image; however, our results indicate this is not the only mechanism that motivates the balance conversation in other social mirrors seen in prior work. Heavily emphasized conditions saw no reported change in social pressure and only a small change in participation attributed to that distortion.

The two studies bring both practical and theoretical implications to the study of real-time visualization, group dynamics and social computing. Validating two of our three hypotheses, we showed that people would trust a visualization over their own perception and that people would participate less when overemphasized. Though we set out to demonstrate a malleable link between distortion and participation, we found there must be other mechanisms that must account for the push towards balance in conversation. We expect the dominant mechanism for change is the knowledge that one could be held accountable by the visualization rather than by judging the visualization's account directly.

Using abstract visualization as feedback, a distorted visualization produces an effect very near that of an undistorted one. The accuracy of the visualization has a minor impact as long as the visualization remains trustworthy. At $60 \%$ additional contribution participants shifted 2 seconds in individual contribution per minute. Though we did not test visualizations that were so far skewed to be untrustworthy, we would anticipate they would be easily ignored based on our preliminary test with amplitude and the negative feedback the other distortions received.

Though the direct influence of social mirrors on contribution are less clear, the self-reflection and effect predicted by Goffman's theories can still be seen. However, that effect seems to be caused more by participants simply being more self aware rather than through the visualization itself. As seen in previous works, visualizations like the Conversation Clock articulate and help people realize what may not be readily obvious to them or to their conversation group (i.e. "I really have been talking too much," or "I should speak up"). Individuals reported altering their behavior based on what was seen in the visualization because it reflected on themselves, perhaps they altered their behavior based on what they knew to be true and simply confirming it in the visualization.

People deferred to the visualization over their own judgement of conversation as shown in the pilot study. As a motivational tool, the use of distorted feedback could be an effective means to challenge people in achieving a targeted contribution goal. Work in physical therapy has been able to appropriate similar feedback to encourage recovering patients to push their muscles harder by underreporting their performance [6]. Though the work presented here was done with participants without a specific motivation to alter their interaction, a similar approach could be taken to use social pressure as a motivation: teaching social skills directly in conversation, conserving energy compared to one's neighbors, or increasing exercise relative to one's friends. Distorted feedback could help to drive individuals when there is a goal.

In the second study, we found the social mirror was most useful at a specific point in time. The Conversation Clock played a role in between questions at roughly 6-8 minutes into the conversation, a time when 
participants took a moment to break from speaking and switch topics. Past work also indicates the clock is most useful when one is less actively engaged at a specific moment in the conversation (not speaking or returning eye-contact) [22]. Future work might further study moments of social mirror utility to gauge if a person is watching others or specifically checking themselves. The change occurring between questions in the dyadic condition seems natural as both participants are engaged throughout the remainder of the conversation. Though an extensive categorization of the gaze direction in socially mirrored environments has yet to be done, it could show when people are most interested in feedback and potentially why.

Viewing personal social data alongside others' personal data reveals nuances of personality. Upon viewing that data, context determines how a person responds: assimilate into the crowd, to stand out as an individual, or simply know where they fit. A person can shape themselves based on what aspects they value in that context. Social mirrors have explored a subset of applications in meetings, conferences, the workplace, and therapy; they show that feedback can be used to promote characteristics that are desirable in the group. As more social interactive data becomes accessible with sensors and personal data collection, the settings for social mirror feedback only expands.

\section{Limitations}

Our work is limited to the exploration of the Conversation Clock and our set of distortion strategies in relatively short conversations. We cannot make strong claims as to how this setting affects individuals over long periods of regular use. We expect that once a person is comfortable with the visualization, its effect would decrease unless there were a specific social reason to attend to one's representation. This remains to be seen in future work.

\section{Acknowledgements}

We recognize and thank the Siebel Scholarship Foundation, the UIUC Social Spaces Group, and the UIUC Orchid Group.

\section{References}

[1] Bachour, K., Kaplan, F., and Dillenbourg, P. Reflect; An Interactive Table for regulating face-to-face collaborative learning. Proceedings of EC-TEL. 39-48. 2008.

[2] Bergstrom, T. and Karahalios, K. Conversation Clock: Visualizing audio patterns in co-located groups. Proceedings of HICSS. 2007.

[3] Bergstrom, T. and Karahalios, K. Vote and Be Heard: Adding Back-Channel Cues to Social Mirrors. Proceedings of Interact. 2009.
[4] Bly, S. A., Harrison, S. R., and Irwin, S. Media spaces: bringing people together in a video, audio, and computing environment. Communications of ACM. 1993.

[5] Branigan, H. Perspectives on Multi-party Dialogue. Research on Language \& Computation. 4(153-177. 2006.

[6] Brewer, B. R., Fagan, M., Klatzky, R. L., and Matsuoka, Y. Perceptual limits for a robotic rehabilitation environment using visual feedback distortion. IEEE Transactions on Neural Systems and Rehabilitation Engineering. 13(12005.

[7] DiMicco, J. M. and Bender, W. Group Reactions to Visual Feedback Tools. Persuasive Technology. 2007.

[8] DiMicco, J. M. and Millen, D. R. Identity management: multiple presentations of self in facebook. GROUP. 383386. 2007.

[9] DiMicco, J. M., Pandolfo, A., and Bender, W. Influencing group participation with a shared display. Proceedings of CSCW. 2004.

[10] Donath, J., Karahalios, K., and Viégas, F. Visiphone. Proceedings of ICAD. 2000

[11] Donath, J. and Viégas, F. B. The chat circles series: explorations in designing abstract graphical communication interfaces. Proceedings of DIS. 359-369. 2002.

[12] Ekman, P. 2001 Telling Lies: Clues to Deceit in the Marketplace, Politics, and Marriage. W.W. Norton \& Company.

[13] Erickson, T. and Kellogg, W. A. Social translucence: an approach to designing systems that support social processes. TOCHI. 7(1):59-83. 2000.

[14] Gatica-Perez, D. Automatic nonverbal analysis of social interaction in small groups: A review. Image and Vision Computing. 27(1775-1787. 2009.

[15] Gersick, C. J. G. and Hackman, J. R. Habitual routines in task-performing groups. Organizational Behavior and Human Decision Processes. 47(11990.

[16] Giles, H., Coupland, J., and Coupland, N. 1991 Contexts of Accommodation: Developments in Applied Sociolinguistics. Cambridge University Press.

[17] Goffman, E. 1959 The Presentation of Self in Everyday Life. Doubleday Anchor Books.

[18] Hancock, J. T., Thom-Santelli, J., and Ritchie, T. Deception and design: the impact of communication technology on lying behavior. Proceedings of CHI. 129-134. 2004.

[19] Hare, A. P. Group Size. American Behavioral Scientist. 24(695-709. 1981.

[20] Harry, D., Green, J., and Donath, J. Backchan.nl: integrating backchannels in physical space. Proceedings of CHI. 2009.

[21] Ishii, H. and Kobayashi, M. ClearBoard: a seamless medium for shared drawing and conversation with eye contact. Proceedings of CHI. 525-532. 1992.

[22] Karahalios, K. and Bergstrom, T. Social Mirrors as Social Signals: Transforming Audio into Graphics. IEEE Computer Graphics and Applications. 29(5):22-32. 2009.

[23] Karahalios, K. and Donath, J. Telemurals: linking remote spaces with social catalysts. Proceedings of CHI. 615-622. 2004.

[24] Kim, T., Chang, A., Holland, L., and Pentland, A. S. Meeting mediator: enhancing group collaboration using 
sociometric feedback. Proceedings of CSCW. 457-466. 2008.

[25] Kulyk, O., Wang, J., and Terken, J. Real-Time Feedback on Nonverbal Behaviour to Enhance Social Dynamics in Small Group Meetings. Machine Learning for Multimodal Interaction. 2006.

[26] Lampe, C. A. C., Ellison, N., and Steinfield, C. A familiar face(book): profile elements as signals in an online social network. Proceedings of CHI. 435-444. 2007.

[27] Leshed, G., Perez, D., Hancock, J. T., Cosley, D., Birnholtz, J., Lee, S., McLeod, P. L., and Gay, G. Visualizing real-time language-based feedback on teamwork behavior in computer-mediated groups. 537-546. 2009.

[28] Mehrabian, A. 1971 Silent Messages. Wadsworth.

[29] Olson, G. M. and Olson, J. S. Distance Matters. HumanComputer Interaction. 15(2):139 - 178. 2000.
[30] Pedersen, E. R. and Sokoler, T. AROMA: abstract representation of presence supporting mutual awareness. Proceedings of CHI. 51-58. 1997.

[31] Simons, D., Franconeri, S., and Reimer, R. L. Change Blindness in the absence of a Visual Disruption. Perception. 29(1143-1154. 2000.

[32] Simons, D. J. and Chabris, C. F. Gorillas in our midst: Sustained inattentional blindness for dynamic events. Perception. 28(1059-1074. 1999.

[33] Sturm, J., Herwijnen, O. H.-v., Eyck, A., and Terken, J. Influencing social dynamics in meetings through a peripheral display. ICMI '07: Proceedings of the 9th international conference on Multimodal interfaces. 2007.

[34] Tannen, D. 1994 Gender and Discourse. Oxford University Press. 